\title{
Lipocalin-2 is increased in progressive multiple sclerosis and inhibits remyelination \\ OPEN
}

Faiez Al Nimer, MD,

$\mathrm{PhD}$

Christina Elliott, $\mathrm{PhD}$

Joakim Bergman, MSc

Mohsen Khademi, PhD

Ann M. Dring, PhD

Shahin Aeinehband, MSc

Tommy Bergenheim,

$\mathrm{MD}, \mathrm{PhD}$

Jeppe Romme

Christensen, MD, PhD

Finn Sellebjerg, MD,

$\mathrm{PhD}, \mathrm{DMSc}$

Anders Svenningsson,

MD, PhD

Christopher Linington, $\mathrm{PhD}$

Tomas Olsson, MD, PhD

Fredrik Piehl, MD, PhD

Correspondence to

Dr. Al Nimer:

faiez.al.nimer@ki.se

Supplemental data at Neurology.org/nn

\section{ABSTRACT}

Objective: We aimed to examine the regulation of lipocalin-2 (LCN2) in multiple sclerosis (MS) and its potential functional relevance with regard to myelination and neurodegeneration.

Methods: We determined LCN2 levels in 3 different studies: (1) in CSF and plasma from a casecontrol study comparing patients with MS $(n=147)$ with controls $(n=50)$ and patients with relapsing-remitting MS ( $n=75$ ) with patients with progressive MS ( $n=72)$; (2) in CSF and brain tissue microdialysates from a case series of 7 patients with progressive MS; and (3) in CSF at baseline and 60 weeks after natalizumab treatment in a cohort study of 17 patients with progressive MS. Correlation to neurofilament light, a marker of neuroaxonal injury, was tested. The effect of LCN2 on myelination and neurodegeneration was studied in a rat in vitro neuroglial cell coculture model.

Results: Intrathecal production of LCN2 was increased predominantly in patients with progressive MS ( $p<0.005$ vs relapsing-remitting MS) and displayed a positive correlation to neurofilament light ( $p=0.005$ ). Levels of LCN2 in brain microdialysates were severalfold higher than in the CSF, suggesting local production in progressive MS. Treatment with natalizumab in progressive MS reduced LCN2 levels an average of 13\% ( $p<0.0001)$. LCN2 was found to inhibit remyelination in a dose-dependent manner in vitro.

Conclusions: LCN2 production is predominantly increased in progressive MS. Although this moderate increase does not support the use of LCN2 as a biomarker, the correlation to neurofilament light and the inhibitory effect on remyelination suggest that LCN2 might contribute to neurodegeneration through myelination-dependent pathways. Neurol Neuroimmunol Neuroinflamm 2016;3:e191; doi: 10.1212/NXI.0000000000000191

\section{GLOSSARY}

BSA = bovine serum albumin; DIV = days in vitro; EAE = experimental autoimmune encephalomyelitis; INDC = inflammatory neurologic disease controls; $\mathbf{L C N 2}=$ lipocalin-2; $\mathbf{M D}=$ microdialysates; $\mathbf{M O G}=$ myelin oligodendrocyte glycoprotein; $\mathbf{M S}=$ multiple sclerosis; NFL = neurofilament light; PBS = phosphate-buffered saline; PPMS = primary progressive MS; $\mathbf{R R}=$ relative recovery; RRMS = relapsing-remitting MS; SC = symptomatic controls; SMI-31 = phosphorylated neurofilament; SPMS = secondary progressive MS; TNF = tumor necrosis factor.

The recent progress in the understanding of the pathophysiology and therapeutic options in multiple sclerosis (MS) pertains mainly to earlier relapsing-remitting MS (RRMS) stages. Our understanding of later disease stages is much more limited, and there is an urgent need to identify biomarkers of pathophysiologic pathways that can increase our knowledge and possibly lead to the identification of new therapeutic targets. ${ }^{1,2}$

Lipocalin-2 (LCN2) is a $25-\mathrm{kDa}$ protein that was first identified as an acute phase protein stored and secreted by neutrophils. ${ }^{3,4}$ It has now been ascribed multiple signaling roles, such as iron delivery, cell survival/death, differentiation, and inflammation, in physiologic and

From the Neuroimmunology Unit (F.A.N., M.K., S.A., T.O., F.P.), Department of Clinical Neuroscience, Karolinska Institutet, Stockholm, Sweden; Institute of Infection, Immunity and Inflammation (C.E., C.L.), University of Glasgow, UK; Department of Pharmacology and Clinical Neuroscience (J.B., A.M.D., A.S.) and Neurosurgery (T.B.), Umeå University, Sweden; and Danish Multiple Sclerosis Center (J.R.C., F.S.), Department of Neurology, Rigshospitalet, University of Copenhagen, Denmark.

Funding information and disclosures are provided at the end of the article. Go to Neurology.org/nn for full disclosure forms. The Article Processing Charge was paid by the authors.

This is an open access article distributed under the terms of the Creative Commons Attribution-NonCommercial-NoDerivatives License 4.0 (CC BY-NC-ND), which permits downloading and sharing the work provided it is properly cited. The work cannot be changed in any way or used commercially. 
pathologic conditions. ${ }^{5}$ Recently, a number of studies have pointed to a role for LCN2 in the CNS as well; in experimental models and cell culture systems, LCN2 induces reactive astrocytosis, neuronal migration, and death, and it also possibly has a detrimental effect on oligodendrocytes. ${ }^{6-10}$ It also promotes M1 polarization of microglia and mediates their deramification and apoptosis. ${ }^{11,12}$ Studies in experimental autoimmune encephalomyelitis (EAE) have suggested functional roles for LCN2, with regulatory effects on disease severity, proliferation of $\mathrm{T}$ cells, and demyelination. ${ }^{13-16}$ Of note, LCN2 has been shown to be increased in a small cohort of patients with progressive disease (compared with RRMS). ${ }^{15}$

Therefore, in a case-control study, we compared the intrathecal production of LCN2 between patients with RRMS, patients with progressive MS, and controls, and the results prompted us to further investigate its regulation and potential functional relevance in progressive MS.

METHODS Study design and patient samples. We determined LCN2 levels in 3 different studies. First, we compared LCN2 production between patients with MS and controls and between patients with RRMS and progressive MS in a casecontrol study. CSF and plasma samples were obtained from an in-house biobank (Karolinska University Hospital, Sweden) containing samples collected during routine neurologic workups from 2003 to 2012. Demographic data of the patients included in this study are presented in table e- 1 at Neurology.org/nn. A total of 197 patients were included, of which 147 were patients with MS fulfilling the McDonald criteria (RRMS = 75 [relapse $=19$, remission = 56]; secondary progressive MS $[$ SPMS $]=49$; primary progressive MS [PPMS] $=23$ ). SPMS was defined as an initial relapsing-remitting disease course followed by more than 12 months of continuous worsening $(\geq 0.5$ Expanded Disability Status Scale point) not explained by relapses. At time of sampling, none of the patients had received immunomodulatory treatment. Control groups were composed of symptomatic controls (SC) $(\mathrm{n}=39$; sensory symptoms $=34$, dizziness $/$ vertigo $=3$, tension headache $=2$ ) and inflammatory neurologic disease controls (INDC) ( $\mathrm{n}=11$; systemic lupus erythematosus $=4$, herpes encephalitis $=2$, sarcoidosis $=1$, anti-NMDA receptor encephalitis $=1$, progressive multifocal encephalopathy $=1$, demyelinating disease of unknown etiology $=1$, myelopathy of unknown etiology $=1$ ) according to the guidelines for biomarker studies in MS. ${ }^{17}$ We used SC and INDC to investigate whether $\mathrm{LCN} 2$ can be used as a biomarker to distinguish between patients with MS and patients with similar neurologic symptoms and patients with similar CSF laboratory parameters, respectively. Second, we determined LCN2 levels in the CSF and microdialysates $(\mathrm{MD})$ in a case series study of 7 patients with SPMS who received intrathecal delivery of rituximab (Umeå University, NCT01719159). In this study, microdialysis catheters were used to monitor the treatment effect. They were inserted at baseline (day 0) in periventricular brain tissue and perfused with Plasmodex solution for 7 days. CSF was collected by lumbar puncture $1-2$ days before the operative procedure and MD were collected 6 times a day. Finally, we measured the CSF levels of LCN2 in an open-label trial of natalizumab in progressive MS. In this cohort, LCN2 was determined in CSF collected from 10 patients with PPMS and 7 patients with SPMS at baseline and after 60 weeks of treatment with natalizumab. The details of the study design and outcome have been previously published. ${ }^{18}$

Standard protocol approvals, registrations, and patient consents. The regional ethical vetting boards of Stockholm (main study; 2003/2-548), Umeå (MD substudy; 2009/210731-2), and Copenhagen (natalizumab substudy; 2012-334$32 \mathrm{M}$ ) approved the study procedures, and written informed consent was obtained from all patients.

Measurements and relative recovery of LCN2 by microdialysis. CSF samples were centrifuged $(300 \mathrm{~g})$ immediately after sampling, aliquoted, and stored at $-80^{\circ} \mathrm{C}$ until analysis. Levels of neurofilament light (NFL) in the main CSF cohort were obtained from a previously published dataset. ${ }^{19} \mathrm{LCN} 2$ levels in CSF and plasma were measured using a commercially available ELISA kit (R\&D Systems, Minneapolis, MN). Because LCN2 values reported in the literature were higher than those measured in our material, ${ }^{14,20}$ we also ran approximately one-seventh of the CSF samples, including all groups of patients except for INDC, on an ELISA kit from Bioporto (Copenhagen, Denmark). The LCN2 levels measured with the Bioporto kit were $30 \%$ higher on average but correlated very well with the levels obtained from the R\&D ELISA $\left(R^{2}=0.93\right)$ and were adjusted to the more conservative estimate. Measurements were optimized and performed for LCN2 using a 1:1.2 CSF to phosphate-buffered saline (PBS) dilution and a 1:200 plasma to PBS dilution. For the MD samples and their matched CSF obtained by lumbar puncture, the Bioporto ELISA was used because of the higher sensitivity and the need to work with higher dilutions because of the smaller collected volumes.

LCN2 is a molecule that exists in high concentrations in the blood, and passive leakage to the CNS may contribute to the levels detected in the intrathecal compartment. Therefore, we calculated the LCN2 index, which likely better reflects the intrathecal production of LCN2, according to the formula used to calculate the IgG index (i.e., LCN2 index: [CSF LCN2/CSF albumin]/ [plasma LCN2/plasma albumin]) and using samples obtained at the same time for each patient. CSF was available for LCN2 measurements from all patients. Patients for whom LCN2 index was not calculated because of random missingness of 1 or more of the other 3 parameters were excluded from the analyses $(S C=2$, INDC $=2$, RRMS $=3$, SPMS $=18$, PPMS $=5$ ).

To estimate the real in vivo brain tissue concentrations of LCN2, the relative recovery (RR) of LCN2 by microdialysis was calculated in an in vitro experiment. Recombinant human LCN2 (Sigma-Aldrich, St. Louis, MO) was used for the RR experiment at concentrations 1 time, 10 times, and 100 times the maximum concentration obtained in the MD samples from patients. Recombinant LCN2 was diluted in Ringer solution with $0.2 \mathrm{mg} / \mathrm{mL}$ bovine serum albumin (BSA) to form an "artificial interstitial fluid" compartment. An MD catheter was immersed in the artificial solution and then perfused by Plasmodex solution by the same MD pump system as in the patients. After flush priming of the catheter, the MD fluid was drained to a waste tube for at least 40 minutes before sampling. Subsequently, MD were collected for 2 lots of 2 hours. The LCN2 concentrations in the 
artificial solution and the collected MD were measured by ELISA (Bioporto).

Myelinating cultures. In vitro rat myelinating cultures were established as described previously. ${ }^{21}$ Cultures were maintained at $37^{\circ} \mathrm{C} / 7 \% \mathrm{CO}_{2}$ and fed thrice weekly by replacing half the culture medium with fresh differentiation media. After 12 days in vitro (DIV), insulin was omitted from the culture medium to promote myelination, with further culturing for up to 30 days. Myelinating cultures were either untreated or treated daily with $10 \mathrm{ng} / \mathrm{mL}$, $100 \mathrm{ng} / \mathrm{mL}$, or $1,000 \mathrm{ng} / \mathrm{mL}$ of recombinant rat LCN2 (R\&D Systems) from 18 DIV (early) or 24 DIV (late) and for 10 or 6 days, respectively. Experiments were performed 3 times.

Immunochemistry. The following antibodies were used: mouse monoclonal SMI-31 (phosphorylated neurofilament, Abcam, Cambridge, UK), Z2 (anti-MOG [myelin oligodendrocyte glycoprotein]), ${ }^{22}$ and rabbit polyclonal NG2 (chondroitin sulphate proteoglycan, Millipore, Billerica, MA). Secondary antibodies were labeled with Alexa Fluor 488 or Alexa Fluor 555 (Invitrogen, Waltham, MA). To visualize extracellular epitopes on live cells, primary antibody was applied for 30 minutes at $4^{\circ} \mathrm{C}$. After repeated washing in ice-cold Dulbecco's modified eagle medium, subsequent steps were at room temperature. Cells were fixed in $4 \%$ paraformaldehyde for 20 minutes at room temperature. For cytoplasmic antigens, cells were permeabilized with $0.5 \%$ Triton X-100/PBS for 10 minutes (Sigma-Aldrich) followed by 1 hour in $1 \% \mathrm{BSA} / 10 \%$ normal goat serum/0.3M glycine. This was followed by application of primary antibodies for 1 hour, repeated PBS washing, application of appropriate secondary antibodies for 15 minutes, washing with PBS and distilled $\mathrm{H}_{2} \mathrm{O}$, and mounting in Vectashield (Vector Laboratories, Burlingame, CA).

Image analysis. In each case, a minimum of 10 images (10X magnification) were acquired from 3 coverslips using an Olympus $\mathrm{B} \times 51$ fluorescent microscope and Image-Pro software (Media Cybernetics, Rockville, MD). Axonal density was quantified using ImageJ software (NIH systems, version 1.41) as the relative area positive for SMI-31 (SMI-31+). To calculate the percentage of myelinated axons, MOG immunoreactive $\left(\mathrm{MOG}^{+}\right)$myelin sheaths were determined using the BRAINS BATCH algorithm, which uses pattern recognition software to distinguish between linear myelinated internodes and oligodendrocyte cell bodies. To quantify cell numbers, a minimum of 30 images were taken from 3 coverslips (20× magnification) and staining density was quantified using ImageJ. Cell counts were expressed as a percentage of the total $\mathrm{NG} 2+$ pixels within the total field.

Statistical analyses. Analyses were performed with Microsoft Excel and GraphPad Prism 5.0. Comparisons were done by 1 -way analysis of variance with Bonferroni post hoc test. The mean values were used to calculate fold changes and are shown in the graphs. Correlation analyses were performed using the Pearson test.

RESULTS Intrathecal production of LCN2 is increased in progressive MS and correlates to NFL. Albumin quotient was significantly higher in patients with MS than in SC (1.25-fold), but there was no difference between MS subtypes. Plasma LCN2 was higher in INDC than in both SC and patients with MS (figure 1). The CSF LCN2 levels, as well as the LCN2 index, were higher (1.51- and 1.25- fold, respectively) in patients with MS than in SC (figure 2, A and B). Upon stratification for the MS disease subtypes, we found that the CSF levels and the LCN2 index were higher in patients with SPMS (1.27- and 1.39-fold, respectively) and patients with PPMS (1.32- and 1.27-fold, respectively) than in patients with RRMS, although the LCN2 index comparison between PPMS and RRMS was not statistically significant (figure 2, C and D). The LCN2 index, but not CSF LCN2, was higher in remission than in relapse (figure $2, \mathrm{E}$ and $\mathrm{F}$ ). The higher levels of LCN2 in progressive MS were replicated in a separate cohort of 22 patients with RRMS and 24 patients with SPMS (1.39-fold, $p<$ 0.001 , data not shown). Because we found that LCN2 is increased in progressive MS, we next sought to study a possible functional role of LCN2 in human progressive disease and found that the LCN2 index correlated significantly to NFL (figure 3A). LCN2 did not correlate to age in all MS disease subtypes, and NFL levels were higher in RRMS than in SPMS (data not shown).

LCN2 is found in high concentrations in in vivo brain tissue and its production is modestly reduced by natalizumab. We next investigated whether the higher LCN2 levels reflect a local production in the CNS in progressive disease and whether LCN2 is regulated by adaptive immune pathways. Determination of LCN2 levels in MD collected at day 1, 2, and 3 after placement of MD catheters revealed much higher levels than those found in CSF from the same patients. $\mathrm{RR}$ as calculated by the in vitro experiment was $9 \%$ on average. This is similar to the RR measured in in vitro recovery studies with the $100-\mathrm{kDa}$ cutoff MD membranes used for other molecules with similar molecular weights. ${ }^{23}$ Collectively, the estimated local brain LCN2 concentrations were much higher than those in CSF, ranging from 13 times to 853 times the CSF values, which corresponds to concentrations in the range of $12-$ $656 \mathrm{ng} / \mathrm{mL}$ (table 1).

Determination of LCN2 levels in patients with PPMS and SPMS participating in an open-label study with natalizumab ${ }^{18}$ revealed a decrease of LCN2 60 weeks after treatment, but only by $13.9 \%$ on average (figure 3B).

LCN2 inhibits myelination in neuroglial cell cocultures. Because demyelination and impaired remyelination are important characteristics of MS pathophysiology, we next studied the effect of LCN2 in myelinating cultures. Addition of $100 \mathrm{ng} / \mathrm{mL}$ of LCN2 at 18 DIV inhibited myelination by $42.4 \%$, whereas addition of $1,000 \mathrm{ng} / \mathrm{mL}$ resulted in a $78.9 \%$ inhibition (figure 4A). In contrast, exposure to $1,000 \mathrm{ng} / \mathrm{mL}$ of LCN2 at a later stage (24-30 DIV), when myelination in the cocultures had already occurred, did not affect myelination (figure 4B). LCN2 did not have 
A

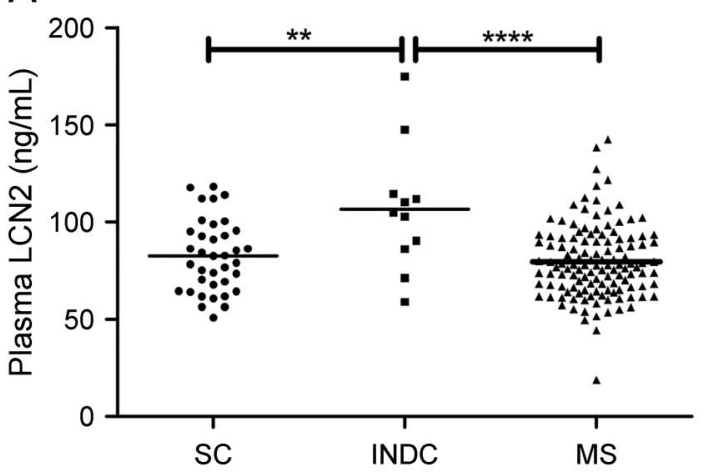

C

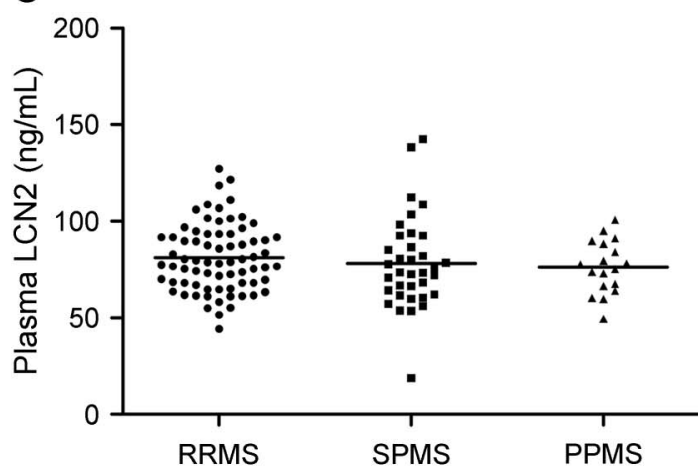

$\mathrm{E}$

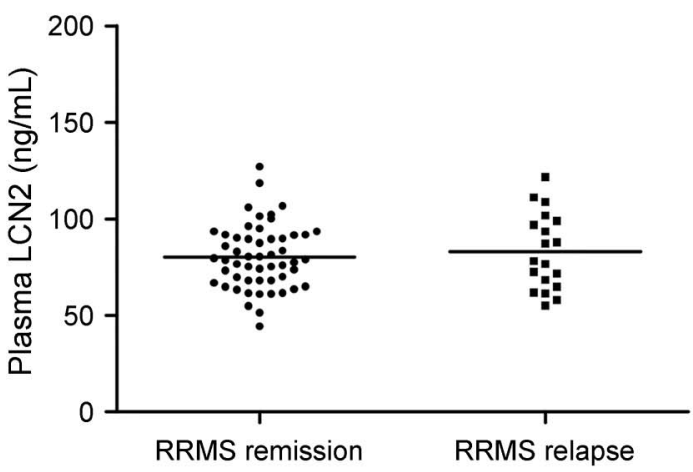

B

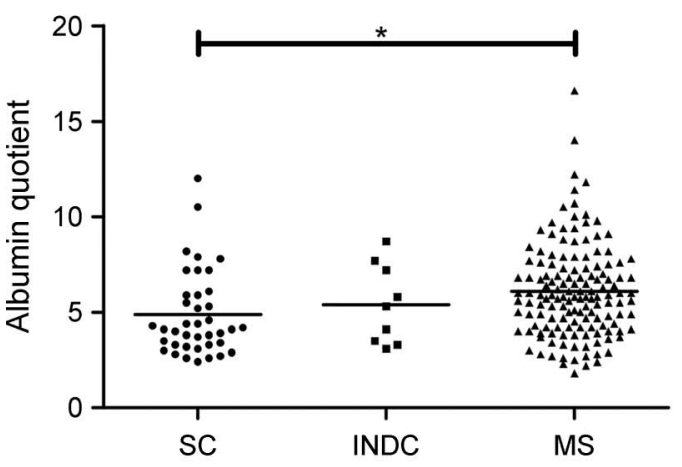

D

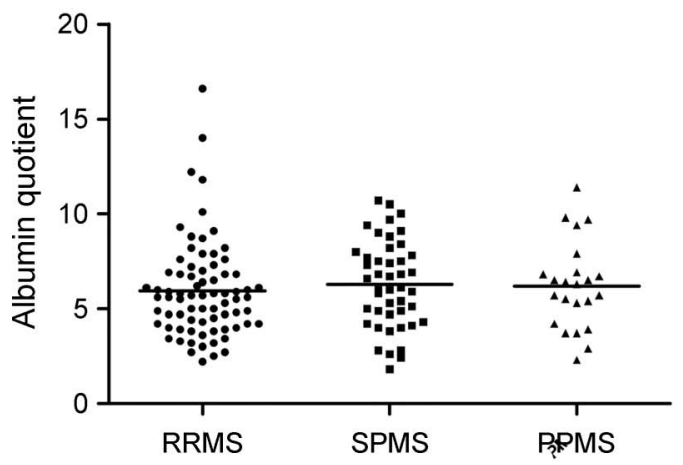

F

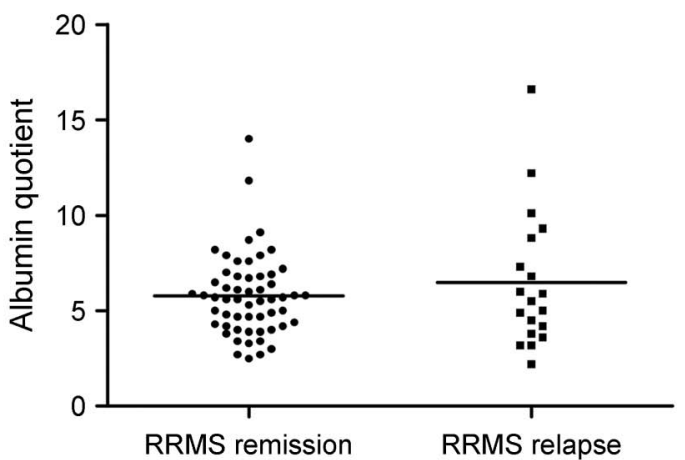

Plasma levels of lipocalin-2 (LCN2) and albumin quotient in (A, B) symptomatic controls (SC), inflammatory neurologic disease controls (INDC), and patients with multiple sclerosis (MS); in (C, D) patients with relapsing-remitting MS (RRMS), secondary progressive MS (SPMS), and primary progressive MS (PPMS); and in (E, F) patients in RRMS remission and RRMS relapse. $* p<0.05, * * p<0.01$, and $* * * * p<0.0001$.

any effect on oligodendroglial progenitor cell numbers or axonal density (figure e-1).

DISCUSSION Because EAE studies suggested that LCN2 is a potentially important molecule for CNS autoimmunity, a biomarker for MS, and increased in the progressive form of MS, we measured LCN2 in a large case-control study of patients with MS and controls and found CSF LCN2 levels and intrathecal LCN2 production to be increased in patients with MS, predominantly those with progressive disease. This finding was subsequently replicated in an independent cohort of patients.
Our data are in accordance with the increased CSF LCN2 levels but not the increased plasma LCN2 levels reported previously in much smaller cohorts. $^{14,15}$ However, plasma LCN2 levels were increased in INDC, in line with previous publications. ${ }^{24,25}$ However, the increase of the LCN2 index in patients with MS compared to controls and in progressive disease compared to RRMS is relatively moderate, with an overlap between the groups. This suggests that LCN2 is likely not a suitable biomarker for clinical diagnosis and prognosis; rather, its increase and correlation to NFL in SPMS indicate a functional role in this 

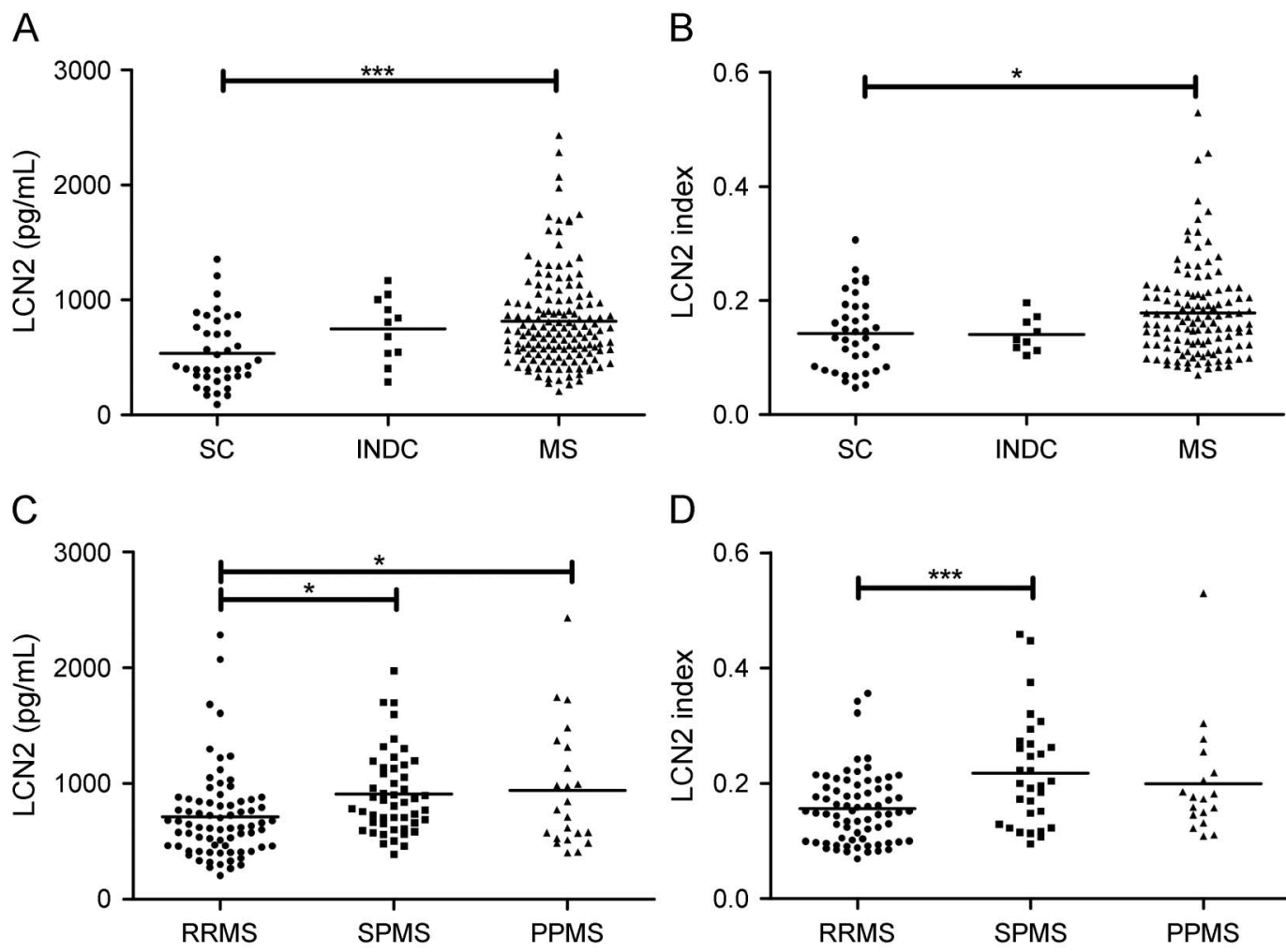

$\mathrm{D}$
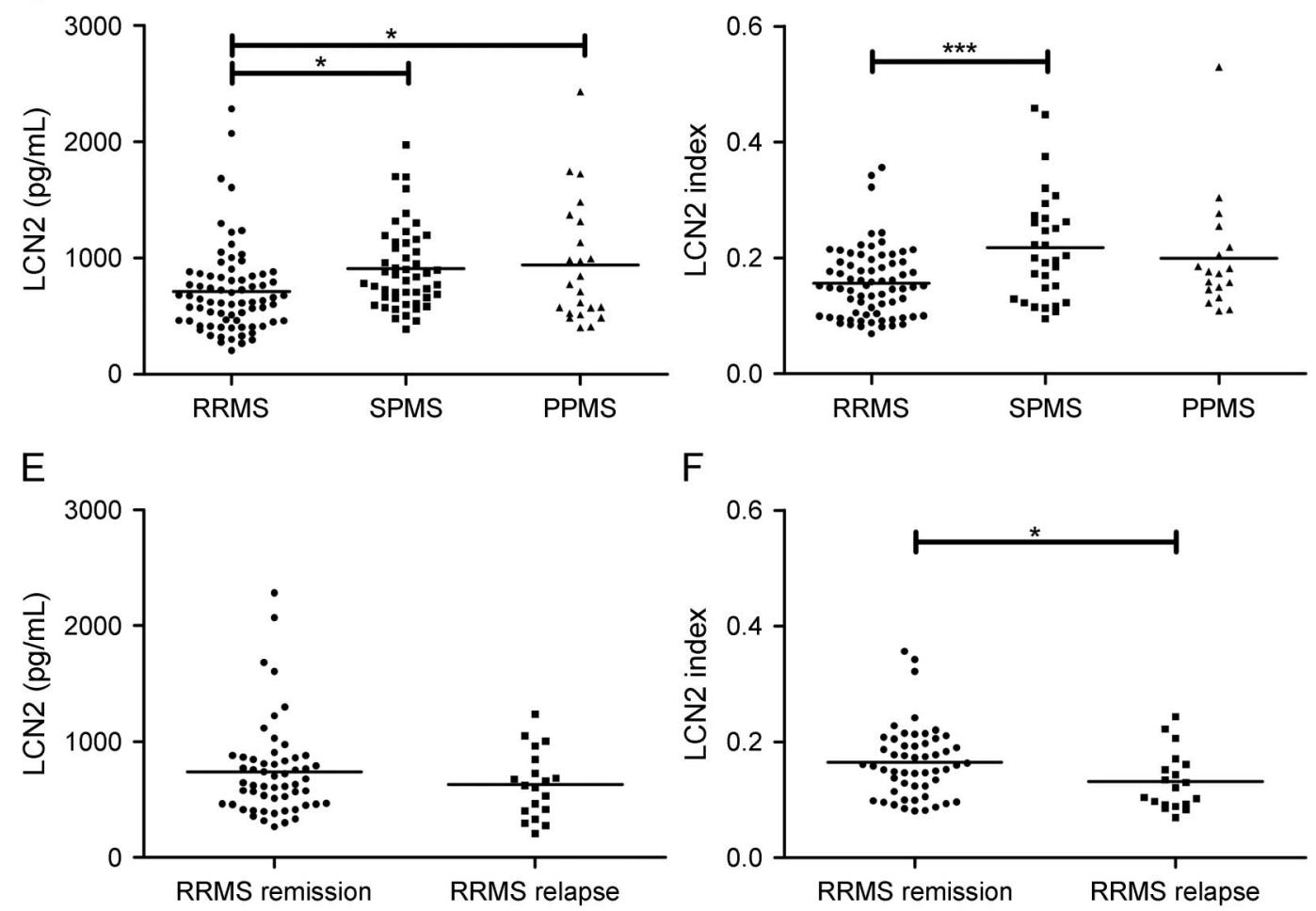

$\mathrm{F}$

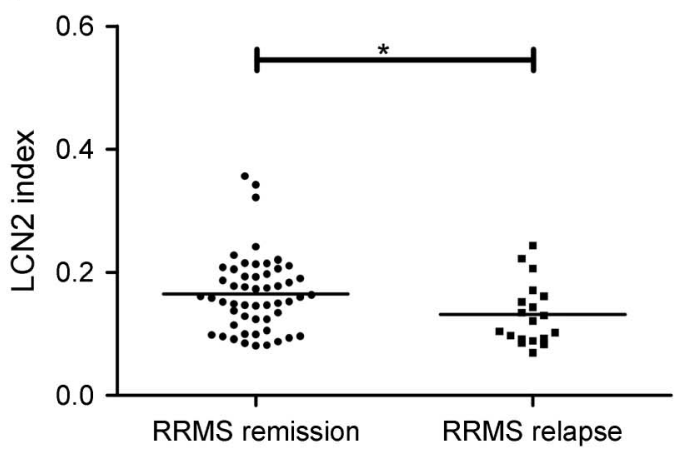

CSF levels of lipocalin-2 (LCN2) and LCN2 index values in (A, B) symptomatic controls (SC), inflammatory neurologic disease controls (INDC), and patients with multiple sclerosis (MS); in (C, D) patients with relapsing-remitting MS (RRMS), secondary progressive MS (SPMS), and primary progressive MS (PPMS); and in (E, F) patients in RRMS remission and RRMS relapse. $* p<0.05$ and $* * * p<0.001$.

disease form. NFL is an axonal protein and a marker that is increased during all disease stages in MS; it is highest during relapses but is also increased in progressive disease and correlates with disease activity. ${ }^{26,27}$ Therefore, the correlation between LCN2 and NFL is interesting because it is observed in a disease stage for which our knowledge about pathogenic processes is still limited. A detrimental effect of LCN2 on axonal degeneration in progressive MS can be induced by several mechanisms, as LCN2 has been shown to modulate CNS inflammation, induce neuronal cell death, regulate dendritic spine formation, and, importantly, modulate iron availability and transfer to the cells, which has been suggested to be of particular importance for demyelination and neurodegeneration in progressive MS. ${ }^{6,13,20,28-31}$

We also describe an effect of LCN2 in inhibiting remyelination, but not inducing demyelination, in in vitro cell cultures in concentrations corresponding to in vivo brain tissue levels of 5 of 7 patients with progressive disease. This finding suggests that LCN2 might induce neurodegeneration through myelination-dependent pathways, a mechanism that has been well described in MS in previous studies. ${ }^{32,33}$ Even though axonal density in cocultures was not affected by inhibition of remyelination through LCN2, it should be noted that it is difficult to translate acute treatment data in an experimental in vitro 

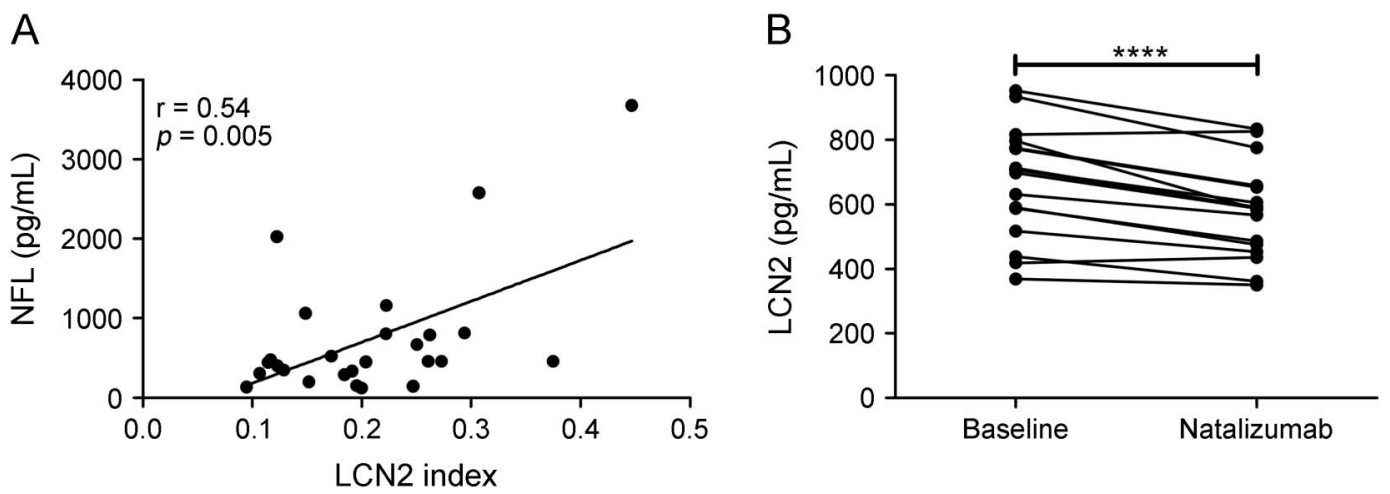

(A) Correlation between neurofilament light (NFL) and lipocalin-2 (LCN2) index in secondary progressive multiple sclerosis (SPMS). (B) Effect of 6 months of treatment with natalizumab on CSF LCN2 levels in progressive multiple sclerosis. $* * * * p<0.0001$.

model to chronic exposure over extensive time periods in the human brain tissue in vivo. The fact that LCN2 might inhibit remyelination is also important because impaired remyelination is a major component of MS pathology for which there are currently no good biomarkers or therapeutic options, although such treatments are in early stages of clinical trials.

In terms of the cause of the increased LCN2 levels in progressive MS, recent studies suggest a shift to a Th17-mediated response and also higher levels of tumor necrosis factor (TNF) in progressive MS, while both interleukin-17 and TNF may drive expression of LCN2 through effects on its promoter. ${ }^{34-37}$ In an effort to further study the production of LCN2 and especially to dissect its regulation by adaptive vs innate immune pathways, we observed that treatment with natalizumab, which effectively targets lymphocyte migration into the CNS and drastically reduces NFL levels in RRMS, ${ }^{38}$ had only a limited effect in terms of reducing LCN2 CSF levels. This finding suggests that production of LCN2 is in large part independent of adaptive immune responses and also highlights pathways of regulation other than those suggested by EAE studies, in which natalizumab drastically reduced LCN2 production in the CNS. ${ }^{14} \mathrm{Neu}-$ trophils may also play a key role in this context, because neutrophils are a source of LCN2 and are not blocked by natalizumab. In experimental models, neutrophils play a significant role in clinical onset of EAE, whereas their role in MS, although not clarified in detail, is suggested to be more prominent in later disease stages. ${ }^{39}$ On the other hand, our data are in accordance with recent reports on experimental CNS disease models and human neuropathologic studies that show that infiltrating monocytes/macrophages and neutrophils, as well as astrocytes and neurons (but not lymphocytes), produce LCN2 $2{ }^{6,14,15,20}$ It will thus be important for future studies to investigate the immune cell or molecular pathways that regulate LCN2 in progressive disease to disclose additional pathophysiologic mechanisms that differ from RRMS,

To further investigate the regulation of LCN2 in vivo in progressive MS, we measured LCN2 levels in MD and CSF and found them to be severalfold higher in brain interstitial fluid than in CSF in all 7 patients. In our MD measurements, we included 2 different time points from patient 2 (days 0 and 3) and patient 3 (days 1 and 3 ) and observed a temporal increase and decrease of LCN2 levels, respectively. This observed difference in kinetics possibly indicates that high local tissue production (not serum leakage and/or catheter-induced trauma) is the cause of high LCN2 levels. This is also supported by the fact that

Table 1 LCN2 is severalfold higher in the in vivo brain tissue as seen by comparison of LCN2 levels in MD vs CSF

Patient 1 Patient 2 Patient 2 Patient 3 Patient 3 Patient 4 Patient 5 Patient 6 Patient 7

$\begin{array}{llllllllll}\text { Days after operation } & 1 & 0 & 3 & 1 & 3 & 3 & 2 & 1 & 1 \\ \text { MD LCN2 } & 59,058 & 3,485 & 15,588 & 9,933 & 2,054 & 46,186 & 56,203 & 1,051 & 5,915 \\ \text { (MD LCN2)/RR } & 656,139 & 38,719 & 173,186 & 110,359 & 22,823 & 513,127 & 624,414 & 11,675 & 65,718 \\ \text { LP CSF LCN2 } & 769 & 1,734 & & 1,597 & & 1,269 & 1,150 & 907 & 913\end{array}$

Abbreviations: $\mathrm{LCN2}=$ lipocalin-2; $\mathrm{LP}$ CSF $=\mathrm{CSF}$ obtained via lumbar puncture; $\mathrm{MD}=$ microdialysates; $\mathrm{RR}=$ relative recovery. 

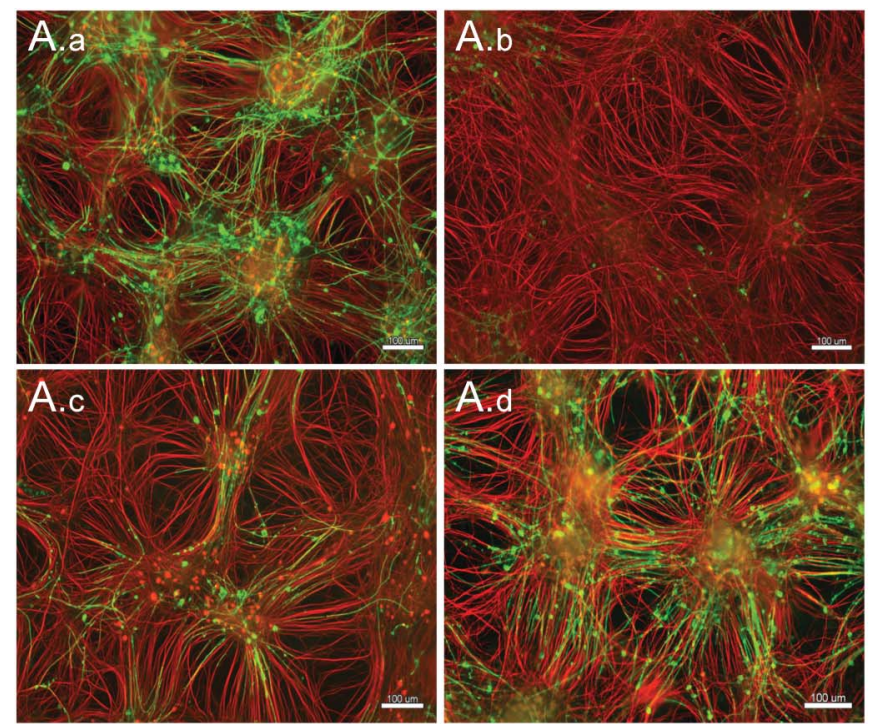

\section{B}
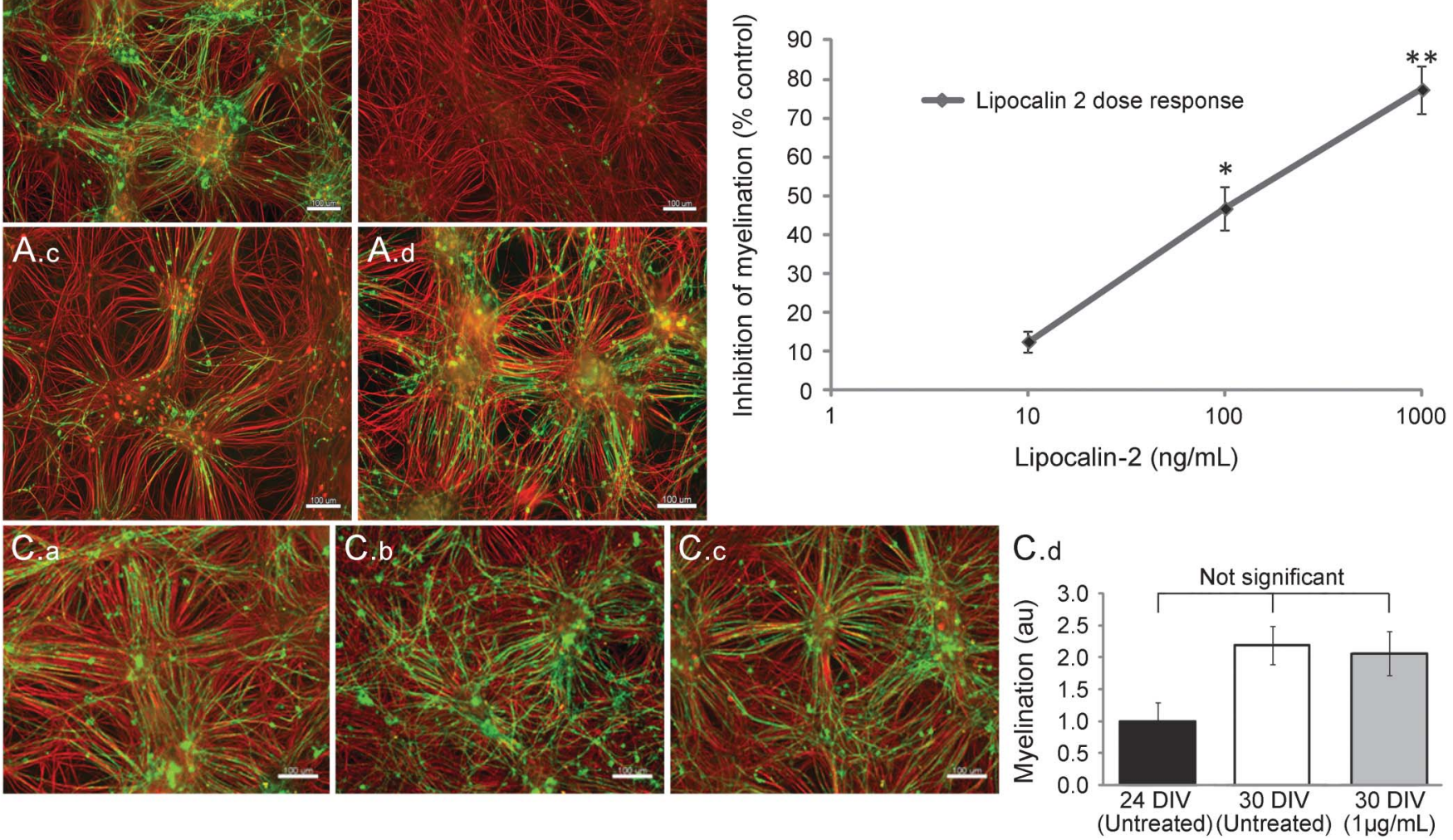

(A) Representative images taken from untreated myelinating cultures (A.a) or after treatment with 1,000, 100, or $10 \mathrm{ng} / \mathrm{mL}$ of lipocalin-2 (LCN2) (A.b-A.d, respectively) with quantification of immunochemical data (B). Untreated myelinating cultures at 24 days in vitro (DIV) (C.a), 30 DIV (C.b), or after addition of $1 \mu \mathrm{g} / \mathrm{mL}$ LCN2 at 24-30 DIV (C.c) with quantification of immunochemical data (C.d.). phosphorylated neurofilament: red; myelin oligodendrocyte glycoprotein: green; scale bar $=100 \mu \mathrm{m} . * p<0.05, * * p<0.01$.

the estimated MD LCN2 levels in some patients were severalfold higher than the serum values measured in the larger cohort. Because studies that have reported the levels of a molecule in both brain interstitial fluid and CSF in MS are rare, more research is needed to further explore the relationship between brain interstitial fluid and CSF in MS for LCN2 and other molecules and/or biomarkers. However, regarding LCN2, the severalfold higher levels in the interstitial fluid are not surprising in light of experimental data showing high local expression in the CNS and the described autocrine functions, and suggest that LCN2 is produced, secreted, and used in the brain tissue, with only a small fraction circulating in the CSF. $711,15,40$

We find that LCN2 is predominantly increased in the intrathecal compartment of patients with progressive MS and that this increase reflects a local tissue production, as indicated by the MD measurements in in vivo brain tissue. Furthermore, LCN2 inhibits remyelination in vitro and correlates to neurodegeneration in SPMS. These observations imply a detrimental role of $\mathrm{LCN} 2$ in progressive MS, where it is locally produced in the CNS and might induce axonal degeneration, possibly through inhibition of remyelination. More studies in progressive disease models and/or MS to further elucidate the pathways that modulate the regulation and effect of LCN2 in progressive MS are warranted.

\section{AUTHOR CONTRIBUTIONS}

F. Al Nimer contributed to the design of the study, data collection, analysis and interpretation of the data, and drafting and writing the manuscript. C. Elliott contributed to the design of the study, data collection, analysis and interpretation of the data, and drafting and writing the manuscript. J. Bergman contributed to data collection and analysis and interpretation of the data. M. Khademi contributed to the design of the study, analysis and interpretation of the data, and revising the manuscript for intellectual content. A.M. Dring contributed to the design of the study, data collection, and analysis and interpretation of the data. S. Aeinehband contributed to data collection and analysis and interpretation of the data. T. Bergenheim contributed to the design of the study and analysis and interpretation of the data. J.R. Christensen contributed to the design of the study and analysis and interpretation of the data. F. Sellebjerg contributed to the design of the study and analysis and interpretation of the data. A. Svenningsson contributed to the design of the study, analysis and interpretation of the data, and drafting and revising the manuscript. C. Linington contributed to the design of the study, analysis and interpretation of the data, and drafting and revising the manuscript. T. Olsson contributed to the design of the study, analysis and interpretation 
of the data, and revising the manuscript. F. Piehl contributed to the design of the study, analysis and interpretation of the data, and drafting and revising the manuscript.

\section{STUDY FUNDING}

This work was supported by the Swedish Research Council (2011-351486774-24), the Swedish Brain Foundation, Knut and Alice Wallenberg Foundation, the Swedish Association of Persons with Neurological Disabilities, and the AFA foundation. F.S. and J.R.C. were supported by the Danish Multiple Sclerosis Society, the Danish Council for Strategic Research, and Brdr. Rønje Holding. A.S. was supported by the Swedish National Multiple Sclerosis Society. C.L. was supported by the United Kingdom Multiple Sclerosis Society. The funders had no role in study design, data collection and analysis, decision to publish, or preparation of the manuscript.

\section{DISCLOSURE}

F. Al Nimer received travel funding from Biogen and Novartis and received research support from Swedish Association of Persons with Neurological Disabilities and Swedish Society for Medical Research. C. Elliott, J. Bergman, M. Khademi, A.M. Dring, and S. Aeinehband report no disclosures. T. Bergenheim is on the editorial board for Journal of Neurooncology and received research support from Swedish Cancer Society. J.R. Christensen received travel funding and/or speaker honoraria from Merck Serono, Biogen, Teva, and Novartis and has consulted for Biogen and Teva. F. Sellebjerg is on the scientific advisory board for Biogen, Genzyme, Merck Serono, Sanofi-Aventis, Teva, and Novo Nordisk; received travel funding and/or speaker honoraria form Bayer Schering, Biogen, Genzyme, Merck Serono, Novartis, Sanofi-Aventis, Schering-Plough, and Teva; has consulted for Biogen; and received research support from Biogen Idec, Sanofi-Aventis, Novartis, Danish Strategic Research Council, Danish Multiple Sclerosis Society, and Lounkaer Foundation. A. Svenningsson served on the advisory board for Sanofi-Genzyme and received travel funding and/ or speaker honoraria from Biogen, Sanofi-Genzyme, Novartis, and Baxter Medical. C. Linington received research support from Multiple Sclerosis Society (UK), Wellcome Trust, and Hertie Stiftung. T. Olsson served on the scientific advisory boards for Merck Serono, Biogen Idec, Genzyme/ Sanofi-Aventis, and Novartis; received travel funding and/or speaker honoraria from Novartis, Biogen Idec, Sanofi-Aventis, Merck, Genzyme, and Medimmune; was coeditor for Current Opinion in Immunology; and received research support from Merck, Biogen, Genzyme/Sanofi-Aventis, Bayer, Novartis, AstraZeneca, the Swedish Research Council, Euratrans Neuroinox, combiMS, Swedish Brain Foundation, AFA Foundation, Knut and Alice Wallenberg Foundation, and Bayer Schering. F. Piehl is on the scientific advisory board for Parexel/Chugai and received research support from Biogen, Novartis, and Swedish Medical Research Council. Go to Neurology. org/nn for full disclosure forms.

Received August 18, 2015. Accepted in final form October 23, 2015.

\section{REFERENCES}

1. Hauser SL, Chan JR, Oksenberg JR. Multiple sclerosis: Prospects and promise. Ann Neurol 2013;74:317-327.

2. Dutta R, Trapp BD. Relapsing and progressive forms of multiple sclerosis: insights from pathology. Curr Opin Neurol 2014;27:271-278.

3. Kjeldsen L, Johnsen AH, Sengelov H, Borregaard N. Isolation and primary structure of NGAL, a novel protein associated with human neutrophil gelatinase. J Biol Chem 1993;268:10425-10432.

4. Kjeldsen L, Bainton DF, Sengelov H, Borregaard N. Identification of neutrophil gelatinase-associated lipocalin as a novel matrix protein of specific granules in human neutrophils. Blood 1994;83:799-807.

5. Chakraborty S, Kaur S, Guha S, Batra SK. The multifaceted roles of neutrophil gelatinase associated lipocalin (NGAL) in inflammation and cancer. Biochim Biophys Acta 2012;1826:129-169.
6. Rathore KI, Berard JL, Redensek A, et al. Lipocalin 2 plays an immunomodulatory role and has detrimental effects after spinal cord injury. J Neurosci 2011;31:13412-13419.

7. Bi F, Huang C, Tong J, et al. Reactive astrocytes secrete $\operatorname{lcn} 2$ to promote neuron death. Proc Natl Acad Sci U S A 2013;110:4069-4074.

8. Zamanian JL, Xu L, Foo LC, et al. Genomic analysis of reactive astrogliosis. J Neurosci 2012;32:6391-6410.

9. Lee S, Park JY, Lee WH, et al. Lipocalin-2 is an autocrine mediator of reactive astrocytosis. J Neurosci 2009;29: 234-249.

10. Kim H, Lee S, Park HC, Lee WH, Lee MS, Suk K. Modulation of glial and neuronal migration by lipocalin-2 in zebrafish. Immune Netw 2011;11:342-347.

11. Lee S, Lee J, Kim S, et al. A dual role of lipocalin 2 in the apoptosis and deramification of activated microglia. J Immunol 2007;179:3231-3241.

12. Jang E, Lee S, Kim JH, et al. Secreted protein lipocalin-2 promotes microglial M1 polarization. FASEB J 2013;27: 1176-1190.

13. Nam Y, Kim JH, Seo M, et al. Lipocalin-2 deficiency ameliorates experimental autoimmune encephalomyelitis: the pathogenic role of lipocalin-2 in the central nervous system and peripheral lymphoid tissues. J Biol Chem 2014;289:16773-16789.

14. Marques F, Mesquita SD, Sousa JC, et al. Lipocalin 2 is present in the EAE brain and is modulated by natalizumab. Front Cell Neurosci 2012;6:33.

15. Berard JL, Zarruk JG, Arbour N, et al. Lipocalin 2 is a novel immune mediator of experimental autoimmune encephalomyelitis pathogenesis and is modulated in multiple sclerosis. Glia 2012;60:1145-1159.

16. Comabella M, Montalban X. Body fluid biomarkers in multiple sclerosis. Lancet Neurol 2014;13:113-126.

17. Teunissen C, Menge T, Altintas A, et al. Consensus definitions and application guidelines for control groups in cerebrospinal fluid biomarker studies in multiple sclerosis. Mult Scler 2013;19:1802-1809.

18. Romme Christensen J, Ratzer R, Bornsen L, et al. Natalizumab in progressive MS: results of an open-label, phase 2A, proof-of-concept trial. Neurology 2014;82:1499-1507.

19. Khademi M, Dring AM, Gilthorpe JD, et al. Intense inflammation and nerve damage in early multiple sclerosis subsides at older age: a reflection by cerebrospinal fluid biomarkers. PLoS One 2013;8:e63172.

20. Naude PJ, Nyakas C, Eiden LE, et al. Lipocalin 2: novel component of proinflammatory signaling in Alzheimer's disease. FASEB J 2012;26:2811-2823.

21. Elliott $\mathrm{C}$, Lindner M, Arthur A, et al. Functional identification of pathogenic autoantibody responses in patients with multiple sclerosis. Brain 2012;135:1819-1833.

22. Piddlesden SJ, Lassmann H, Zimprich F, Morgan BP, Linington $\mathrm{C}$. The demyelinating potential of antibodies to myelin oligodendrocyte glycoprotein is related to their ability to fix complement. Am J Pathol 1993;143:555-564.

23. Helmy A, Carpenter KL, Skepper JN, Kirkpatrick PJ, Pickard JD, Hutchinson PJ. Microdialysis of cytokines: methodological considerations, scanning electron microscopy, and determination of relative recovery. J Neurotrauma 2009;26: 549-561.

24. Rubinstein T, Pitashny M, Levine B, et al. Urinary neutrophil gelatinase-associated lipocalin as a novel biomarker for disease activity in lupus nephritis. Rheumatology (Oxford) 2010;49:960-971. 
25. Kamata M, Tada Y, Tatsuta A, et al. Serum lipocalin-2 levels are increased in patients with psoriasis. Clin Exp Dermatol 2012;37:296-299.

26. Kuhle J, Plattner K, Bestwick JP, et al. A comparative study of CSF neurofilament light and heavy chain protein in MS. Mult Scler 2013;19:1597-1603.

27. Axelsson M, Malmestrom C, Gunnarsson M, et al. Immunosuppressive therapy reduces axonal damage in progressive multiple sclerosis. Mult Scler 2014;20:43-50.

28. Mucha M, Skrzypiec AE, Schiavon E, Attwood BK, Kucerova E, Pawlak R. Lipocalin-2 controls neuronal excitability and anxiety by regulating dendritic spine formation and maturation. Proc Natl Acad Sci U S A 2011; 108:18436-18441.

29. Haider L, Simeonidou C, Steinberger G, et al. Multiple sclerosis deep grey matter: the relation between demyelination, neurodegeneration, inflammation and iron. J Neurol Neurosurg Psychiatry 2014;85:1386-1395.

30. Ropele S, Kilsdonk ID, Wattjes MP, et al. Determinants of iron accumulation in deep grey matter of multiple sclerosis patients. Mult Scler 2014;20:1692-1698.

31. Devireddy LR, Gazin C, Zhu X, Green MR. A cell-surface receptor for lipocalin $24 \mathrm{p} 3$ selectively mediates apoptosis and iron uptake. Cell 2005;123:1293-1305.

32. Trapp BD, Stys PK. Virtual hypoxia and chronic necrosis of demyelinated axons in multiple sclerosis. Lancet Neurol 2009;8:280-291.

33. Kornek B, Storch MK, Weissert R, et al. Multiple sclerosis and chronic autoimmune encephalomyelitis: a comparative quantitative study of axonal injury in active, inactive, and remyelinated lesions. Am J Pathol 2000;157: 267-276.

34. Huber AK, Wang L, Han P, et al. Dysregulation of the IL23/IL-17 axis and myeloid factors in secondary progressive MS. Neurology 2014;83:1500-1507.

35. Rossi S, Motta C, Studer V, et al. Tumor necrosis factor is elevated in progressive multiple sclerosis and causes excitotoxic neurodegeneration. Mult Scler 2014;20: 304-312.

36. Shen F, Ruddy MJ, Plamondon P, Gaffen SL. Cytokines link osteoblasts and inflammation: microarray analysis of interleukin-17- and TNF-alpha-induced genes in bone cells. J Leukoc Biol 2005;77:388-399.

37. Romme Christensen J, Bornsen L, Ratzer R, et al. Systemic inflammation in progressive multiple sclerosis involves follicular T-helper, Th17- and activated B-cells and correlates with progression. PLoS One 2013;8: e57820.

38. Gunnarsson M, Malmestrom C, Axelsson M, et al. Axonal damage in relapsing multiple sclerosis is markedly reduced by natalizumab. Ann Neurol 2011;69:83-89.

39. Rumble JM, Huber AK, Krishnamoorthy G, et al. Neutrophil-related factors as biomarkers in EAE and MS. J Exp Med 2015;212:23-35.

40. Howe CL, Kaptzan T, Magana SM, Ayers-Ringler JR, LaFrance-Corey RG, Lucchinetti CF. Neuromyelitis optica IgG stimulates an immunological response in rat astrocyte cultures. Glia 2014;62:692-708. 


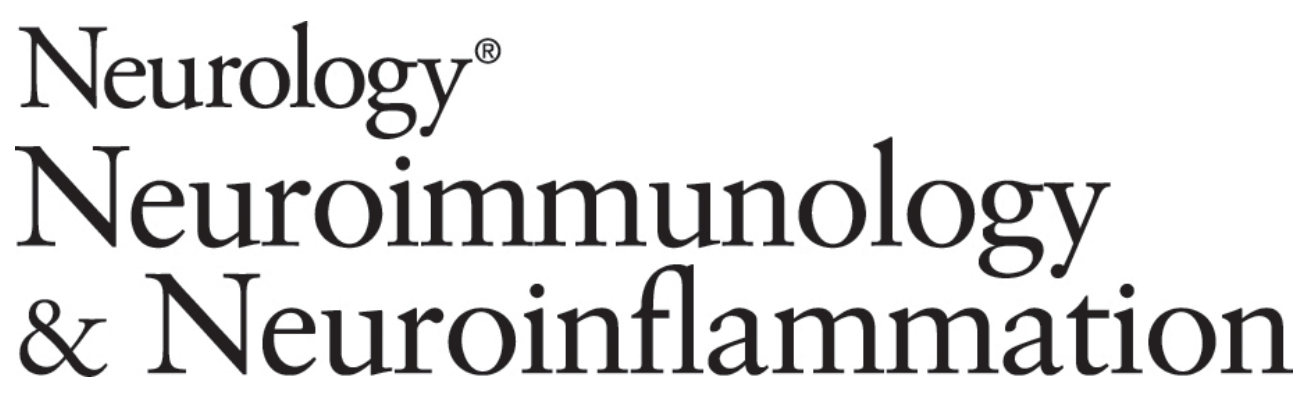

Lipocalin-2 is increased in progressive multiple sclerosis and inhibits remyelination Faiez Al Nimer, Christina Elliott, Joakim Bergman, et al.

Neurol Neuroimmunol Neuroinflamm 2016;3;

DOI 10.1212/NXI.0000000000000191

This information is current as of January 7, 2016

Neurol Neuroimmunol Neuroinflamm is an official journal of the American Academy of Neurology.

Published since April 2014, it is an open-access, online-only, continuous publication journal. Copyright $\odot$ 2016 American Academy of Neurology. All rights reserved. Online ISSN: 2332-7812.

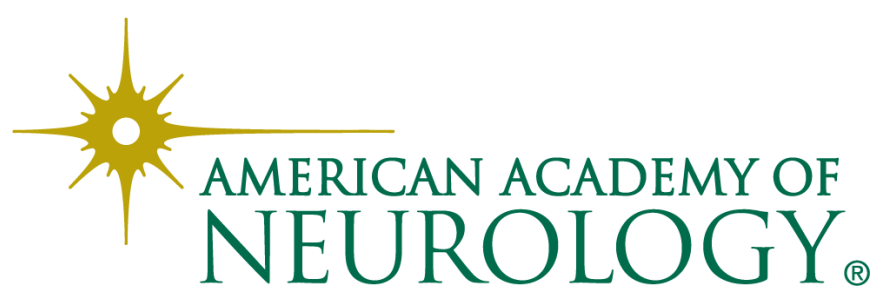




\section{Updated Information \& Services}

\section{Supplementary Material}

\section{References}

Citations

Subspecialty Collections

Permissions \& Licensing

Reprints including high resolution figures, can be found at:

http://nn.neurology.org/content/3/1/e191.full.html

Supplementary material can be found at:

http://nn.neurology.org/content/suppl/2016/01/07/3.1.e191.DC1

This article cites 40 articles, 11 of which you can access for free at: http://nn.neurology.org/content/3/1/e191.full.html\#\#ref-list-1

This article has been cited by 1 HighWire-hosted articles: http://nn.neurology.org/content/3/1/e191.full.html\#\#otherarticles

This article, along with others on similar topics, appears in the following collection(s):

All Demyelinating disease (CNS)

http://nn.neurology.org//cgi/collection/all_demyelinating_disease_cns

Autoimmune diseases

http://nn.neurology.org//cgi/collection/autoimmune_diseases

Cerebrospinal Fluid

http://nn.neurology.org//cgi/collection/cerebrospinal_fluid

Multiple sclerosis

http://nn.neurology.org//cgi/collection/multiple_sclerosis

Information about reproducing this article in parts (figures,tables) or in its entirety can be found online at:

http://nn.neurology.org/misc/about.xhtml\#permissions

Information about ordering reprints can be found online: http://nn.neurology.org/misc/addir.xhtml\#reprintsus

Neurol Neuroimmunol Neuroinflamm is an official journal of the American Academy of Neurology.

Published since April 2014, it is an open-access, online-only, continuous publication journal. Copyright $\odot$ 2016 American Academy of Neurology. All rights reserved. Online ISSN: 2332-7812.

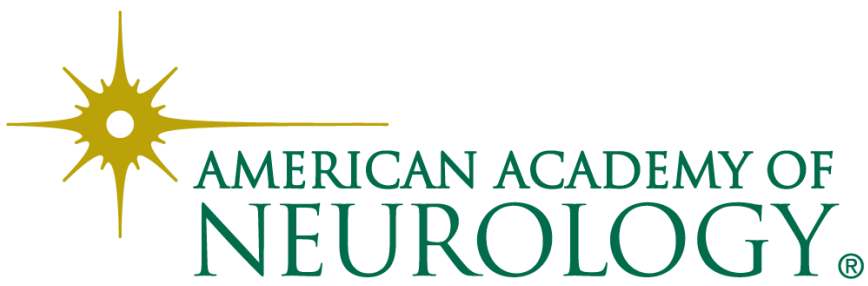

